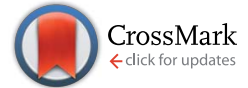

Cite this: Chem. Sci., 2016, 7, 752

\title{
Detection of bioorthogonal groups by correlative light and electron microscopy allows imaging of degraded bacteria in phagocytes $\uparrow$
}

\author{
Daphne M. van Elsland, ${ }^{\text {ab }}$ Erik Bos, ${ }^{c}$ Wouter de Boer, ${ }^{\text {ab }}$ Herman S. Overkleeft, ${ }^{\text {ab }}$ \\ Abraham J. Koster*c and Sander I. van Kasteren*ab
}

\begin{abstract}
The interaction between parasites and phagocytic immune cells is a key inter-species interaction in biology. Normally, phagocytosis results in the killing of invaders, but obligate intracellular parasites hijack the pathway to ensure their survival and replication. The in situ study of these parasites in the phagocytic pathway is very difficult, as genetic modification is often complicated and, if successful, only allows the tracking of pathogen phagocytosis up until the degradation of the engineered reporter constructs. Here we combine bioorthogonal chemistry with correlative light-electron microscopy (CLEM) to follow bacterial processing in the phagolysosomal system. Labelled bacteria are produced using bioorthogonal non-canonical amino tagging (BONCAT), precluding the need for any genetic modification. The bacterial proteome - even during degradation - was then visualised using a novel CLEM-based approach. This allowed us to obtain high resolution information about the subcellular location of the degrading bacteria, even after the proteolytic degradation of reporter constructs. To further explore the potential of CLEM-based imaging of bioorthogonal functionalities, azide-labelled glycans were imaged by this same approach, as well as active-subpopulations of enzymes using a 2-step activity-based protein profiling strategy.
\end{abstract}

Received 6th August 2015

Accepted 21st October 2015

DOI: 10.1039/c5sc02905h

www.rsc.org/chemicalscience
Bioorthogonal chemistry is a powerful tool for labelling of (sub)-populations of biomolecules in complex biological systems ${ }^{7}$ and could be employed to circumvent these problems. The approach relies on the introduction of a small, physiologically inert chemical group into a biomolecule of interest that can subsequently be visualised using a selective reaction. ${ }^{8}$ The small size, biological stability of the chemical group, and the wide range of biomolecules that can be labelled with this approach makes this method a valuable part of the biochemist's toolkit. ${ }^{9,10}$

Bolstered by the recent successful imaging of a pathogen inside a host phagocyte through the use of a bioorthogonally modified cell wall component, D-alanine, ${ }^{11-13}$ we envisaged that bioorthogonally labelled bacteria could also be used to image degradation events in host phagocytes. Bioorthogonal noncanonical amino acid tagging (BONCAT) $)^{14,15}$ for pan-proteomic incorporation of bioorthogonal groups ${ }^{16,17}$ would allow the labelling of a wide range of bacterial species without the need for genetic modification. ${ }^{18}$ Furthermore, unlike reporter proteins, bioorthogonal groups, such as azides ${ }^{19,20}$ have been shown to be stable in the harsh chemical environments of the phagolysosomal system and should therefore be detectable when extensive proteolysis has occurred.

Information about subcellular localization is of key importance when studying parasite-phagocyte-interactions as movement between organelles may be key to the life cycle of certain parasites. $^{1,21}$ Only transmission electron microscopy (TEM)- 


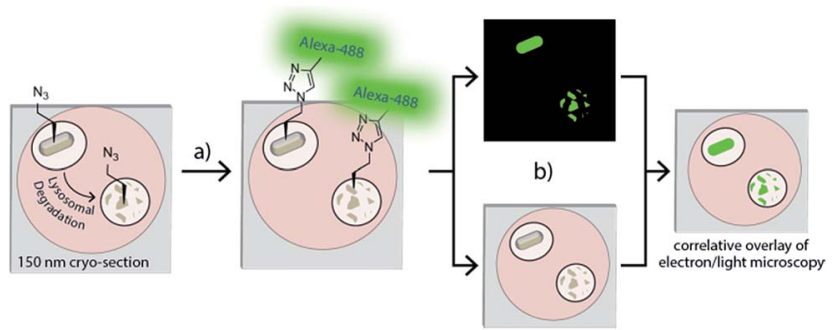

Fig. 1 Overview: (a) phagocytosed azido-E. coli can be fluorescently visualised in an ultrathin cryosection using a ccHc-reaction with a fluorophore; (b) overlay of this image on an electron micrograph provides an ultrastructural context for the signal with nanometreresolution. As the bioorthogonal handle is stable to proteolysis, degraded bacteria can be visualized in this manner.

based techniques allows the study of these pathogens in their subcellular context, as it provides substructural information on the position of any label/antigen within the cell. ${ }^{22}$ However, in contrast to superresolution imaging, ${ }^{23,24}$ no methods have been reported that allow the visualization of bioorthogonal groups using EM-based approaches. ${ }^{25}$

Here we describe the development of a correlative lightelectron microscopy (CLEM)-imaging-based visualisation of bioorthogonal groups that allows imaging of BONCAT-labelled bacteria inside phagocytes (Fig. 1); even as they are being degraded. This approach combines the benefits of confocal microscopy - which allows wide-field navigation to areas of interes $\mathrm{t}^{26}$ - with those of electron microscopy (EM) - which provides narrow field high resolution information about the interior of the cell. ${ }^{22}$ All approaches described here on the model organism E. coli are amenable to application to pathogens, which would open new avenues for studying the events leading to bacterial clearance and/or establishment of intracellular residence by intracellular pathogens.

\section{Results and discussion}

\section{Optimizing the detection of bioorthogonal groups using CLEM}

To develop the CLEM-based imaging of bioorthogonal groups, we initially focussed on azides as our bioorthogonal group of choice, as these are the most versatile of the groups in terms of both incorporation methods and available detection reactions. ${ }^{27,28}$ We used the Met-auxotrophic strain B834(DE3) of $E$. coli as our model bacterium, as it can be readily labelled with azides by metabolic replacement of methionine with azidohomoalanine (Aha). ${ }^{16,29,30}$ However, these labelling strategies can be translated to many other non-auxotrophic bacteria and eukaryotes very effectively. ${ }^{18}$

We first optimised Aha incorporation with respect to cell viability, and incorporation levels into the proteome (Fig. S1†). It was observed that extended incubation times resulted in reduced viability (Fig. S2 $\dagger$ ) and the formation of inclusion bodies (Fig. S3†). This suggests detrimental effects on protein expression and folding of prolonged exposure to Aha. ${ }^{18,31}$ Labelling for $1 \mathrm{~h}$ in presence of $4 \mathrm{mM}$ Aha gave robust signal

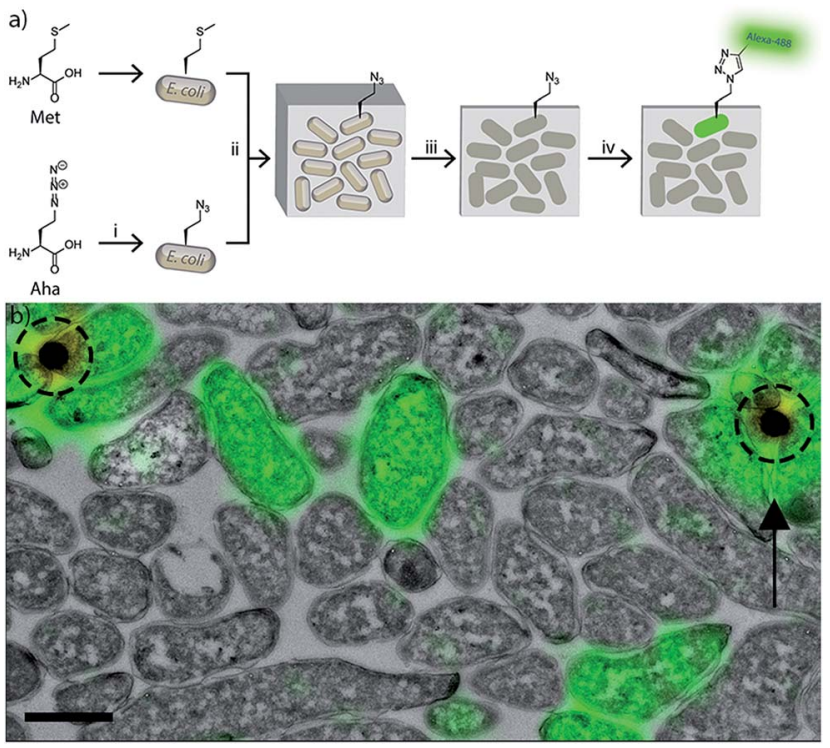

Fig. 2 Optimizing CLEM imaging of azido-E. coli: (a) azido- (i) and unlabelled wt-E. coli were mixed in non-equal ratio (1:25) (ii). After Tokuyasu sample preparation and cryosectioning into $75 \mathrm{~nm}$ sections (iii), a ccHc-reaction with AlexaFluor-488 was performed (iv). (b) CLEMimage of the experiment (a). Green: AlexaFluor-488 label. Dotted circles: signal from the $100 \mathrm{~nm}$ TetraSpeck beads. Scale bar $1 \mu \mathrm{m}$.

and showed minimal inhibition of viability (Fig. S2†). These conditions were used for all further imaging studies.

We focussed our initial development of the on-section labelling of CLEM-samples on cryosections prepared in accordance with Tokuyasu, ${ }^{32}$ as this technique uses mild fixation and sample preparation techniques compatible with azide-chemistry. We prepared sections of mixed azido-E. coli and unlabelled wt- $E$. coli at a non-equal ratio to optimize the on-section bioorthogonal labelling (Fig. 2 and S4†).
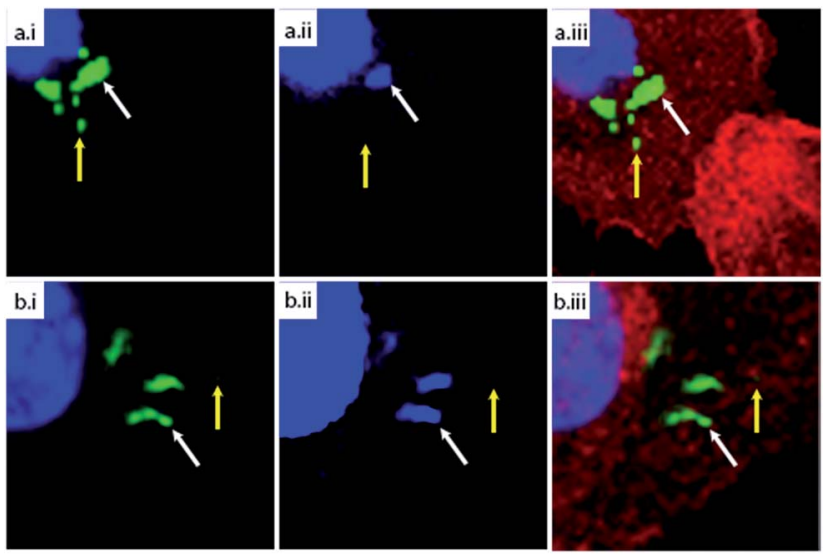

Fig. 3 Confocal microscopy of (a) azido-E. coli or (b) GFP-E. coli after phagocytosis. BM-DCs were pulsed with either azido-E. coli or GFPexpressing $E$. coli ( 45 min pulse). Cells were fixed after a $2 \mathrm{~h}$ chase and stained with DAPI (blue), anti-actin (red) and, in case of azido-E. coli, AlexaFluor-488 alkyne (green = either GFP or AlexaFluor-488). (i) DAPI/488 nm overlay; (ii) DAPI only; (iii) all fluorescent channels overlay. Yellow arrows indicate a 488-single positive focus, white arrows a DAPI/488 $\mathrm{nm}$ double positive focus. 
Of the three available azide-specific bioorthogonal reactions available we focussed on optimising the copper-catalysed Huisgen cycloaddition (ccHc)-reaction ${ }^{8,33,34}$ as it has the lowest background reactivity compared to the strain-promoted cycloaddition reactions, and Staudinger-Bertozzi ligation..$^{35,36}$ Downsides are largely associated with the toxicity of the catalyst. ${ }^{28}$ However, this is not of relevance here as the labelling is performed after aldehydefixation and cryosectioning of the samples. We found that glutaraldehyde-free fixation, followed by a blocking step before the ccHc-reaction, combined with ligand-stabilisation of the $\mathrm{Cu}(\mathrm{I})$ catalytic species gave the best signal-to-noise levels. The addition of aminoguanidine during the ccHc reduced the detrimental effects of the copper sulfate by-products on the DAPI co-staining. ${ }^{37}$

It was also found that care had to be taken when using copper mounting grids on which the ultrathin sections were placed for CLEM-imaging.
If high concentrations of ascorbate and prolonged reaction times were used for the $\mathrm{ccHc}$, we found that the grids dissolved. This could be prevented by shortening reaction times and keeping the ascorbate concentrations low ( $<10 \mathrm{mM})$.

After ccHc-labelling, the sections were first imaged with the confocal microscope (Fig. S4a†) before embedding in methyl cellulose with uranyl acetate. The sections were EM imaged (Fig. S4b $\dagger$ ) and correlation of the confocal and EM images was performed using fluorescent electron-dense beads ${ }^{38}$ as fiducials (Fig. $2 \mathrm{~b}$ and $\mathrm{S} 4 \mathrm{c}-\mathrm{e} \dagger$ ).

\section{Comparison of GFP-E. coli and azido-E. coli for imaging phagolysosomal degradation}

Most CLEM studies employ the fusion of fluorescent proteins to the protein of interest or antibody-based approaches to allow their identification and localisation. ${ }^{22}$ These labelling
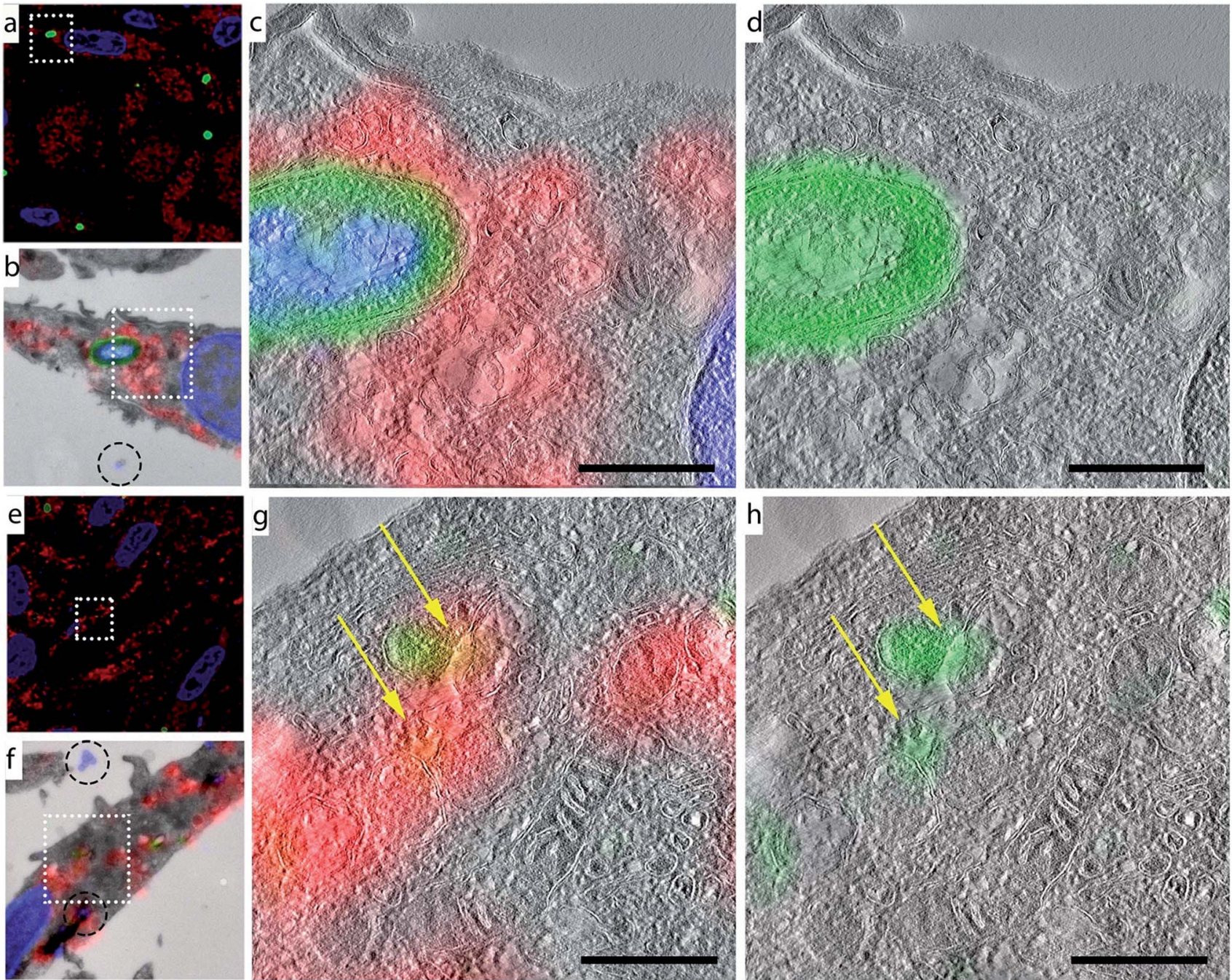

Fig. 4 CLEM imaging of phagocytosed azido-E. coli: BM-DCs were pulsed with azido-E. coli (45 min pulse). Cells were washed with PBS to remove unbound/non-internalized $E$. coli. Samples were fixed immediately after pulsing $(a-d)$ or after a $3 \mathrm{~h}$ chase (e-h). Cells were subjected to Tokuyasu sample preparation and cryosectioned into $150 \mathrm{~nm}$ sections. Sections were reacted with AlexaFluor-488 alkyne using ccHc-conditions (green), anti-LAMP-1 (red) and DAPI (blue). DAPI staining and blue fiducials (indicated with circles in b and f) were used for correlation purposes. (a/e) Confocal microscopy images; (b/f) CLEM image obtained from overlay LM and EM pictures; (c, $d, g$ and h) CLEM details from (b/f), showing LAMP-1 and $488 \mathrm{~nm}$ channels (c/g) or $488 \mathrm{~nm}$ alone (d/h). Scale bar $500 \mathrm{~nm}$. 
approaches have shown to be of great value for the imaging of specific proteins in their cellular context, but only in the cases where genetic modification of the organism has been possible and where the attachment of the fluorescent proteins does not affect protein function. ${ }^{39}$

Immunofluorescence has also been used, but combined with CLEM it either compromises ultrastructure (by virtue of the need of fixation and permeabilisation prior to CLEM-sample preparation), ${ }^{40}$ or suffers from a notoriously low success rate due to compromised epitope availability in samples prepared for TEM. ${ }^{41}$

As our main envisaged application of bioorthogonal CLEM imaging was in detecting phagocytosed bacteria during degradation (Fig. 1), we first determined whether our BONCAT-based approach had advantages over genetic methods for the study of these events. The fate of azido- $E$. coli was compared to that of GFP-expressing $E$. coli (Fig. 3, S5 and S6 $\dagger$ ). We incubated mouse bonemarrow-derived dendritic cells (BM-DCs) $)^{\mathbf{4 2 , 4 3}}$ with azido- $E$. coli or GFP-E. coli for 45 minutes. After washing, the cells were chased for $1 \mathrm{~h}, 2 \mathrm{~h}$ or $3 \mathrm{~h}$ prior to fixation, bioorthogonal modification of the azides (where present), and confocal imaging (Fig. 3 and S5†) - time points in which maturation of a phagosome to a phagolysosome are known to take place in these cells. ${ }^{44}$

To assess whether the fluorescent signal originated from an intact or (partially) degraded bacteria, we made use of extranuclear DAPI staining: colocalisation of the fluorescent signal with the extra-nuclear DAPI indicates the intactness of the bacterial DNA, which in turn indicates the intactness of the bacterium. ${ }^{45}$ Absence of this colocalisation (i.e. $488 \mathrm{~nm}$ single positive foci) indicated the degradation of the bacterial genome and thus death.

The azide-based signal persisted significantly more than the GFP-signal after the killing of the bacterium; as indicated by the significantly larger number of DAPI-negative/azide-positive foci at all time points of the chase period compared to DAPI-negative/GFP-positive foci (Fig. S6†). Many of the azide-positive foci were smaller than intact DAPI/azide double positive foci, indicating these signals to originate from partially degraded bacteria. Control samples of wt- $E$. coli were fully negative at all time points (Fig. S5†).

\section{CLEM-imaging of azido-E. coli after uptake by BM-DCs}

We obtained ultrastructural information about the location of these smaller, DAPI-negative foci by performing CLEM analysis on azido-E. coli-treated BM-DCs samples at all four time points (Fig. 4 and $\mathrm{S} 7 \dagger$ ). Co-staining with the lysosomal marker LAMP-1 revealed that these degraded fragments only partially resided in LAMP-1-positive late endosomes/lysosomes. This ties in with previous studies showing the existence of a second population of phagosomes in DCs, which do not acidify and never become LAMP-1 positive. ${ }^{46}$ This set of phagosomes has been implicated in DC-specific functions such as cross-presentation. ${ }^{47,48}$

Morphological information obtained from TEM showed that the azide-positive/DAPI-positive foci were intact bacteria, whereas the DAPI-negative foci showed no identifiable bacterial morphology, indicating that this technique allows the imaging of partially degraded bacteria inside mammalian phagocytes.

\section{Application of CLEM to other bioorthogonal labelling strategies}

The above approach highlights the potential of CLEM-imaging in detecting BONCAT-labelled bacteria inside phagocytes. However, bioorthogonal chemistry offers a much broader arena of applications to interrogate specific physiological phenomena. ${ }^{27}$ Two stalwart applications are the labelling of glycans in mammalian cells ${ }^{\mathbf{4 9}}$ and activity-based protein profiling. ${ }^{50}$ To firmly embed our technique within the bioorthogonal chemistry paradigm, we also applied CLEM-imaging in these settings.

We first recapitulated the archetypal imaging of sialic acidcontaining glycans on mammalian cells, ${ }^{49}$ by imaging Jurkat cells incubated with $\mathrm{Ac}_{4}-\mathrm{N}$-azidoacetylmannosamine $\left(\mathrm{Ac}_{4}{ }^{-}\right.$ ManNAz; Fig. S8†), an approach that results in cells with a bioorthogonally labelled sialoglycome. ${ }^{51}$ We subjected these cells to the bioorthogonal labelling and imaging conditions as above and found that LM imaging revealed cell surface and intracellular staining as previously observed by Baskin et al. ${ }^{52}$ (Fig. 5a and $\mathrm{S} 9 \dagger)$. Subsequent correlation with EM-imaging of the same section of the cell (Fig. 5b-e) revealed the labelled sialic acids to reside in the Golgi apparatus (Fig. 5d) and the plasma membrane (Fig. 5e). Incubation with unlabelled control $\left(\mathrm{Ac}_{4^{-}}\right.$ ManNAc) gave no fluorescent labelling (Fig. S9a $\dagger$ ).

We next combined CLEM with two-step activity-based protein profiling. ${ }^{53,54}$ This technique has proven powerful for detecting active sub-populations of e.g. serine hydrolases, ${ }^{\mathbf{5 0}}$ cysteine proteases ${ }^{55,56}$ and proteasomes ${ }^{57}$ in a fashion whereby
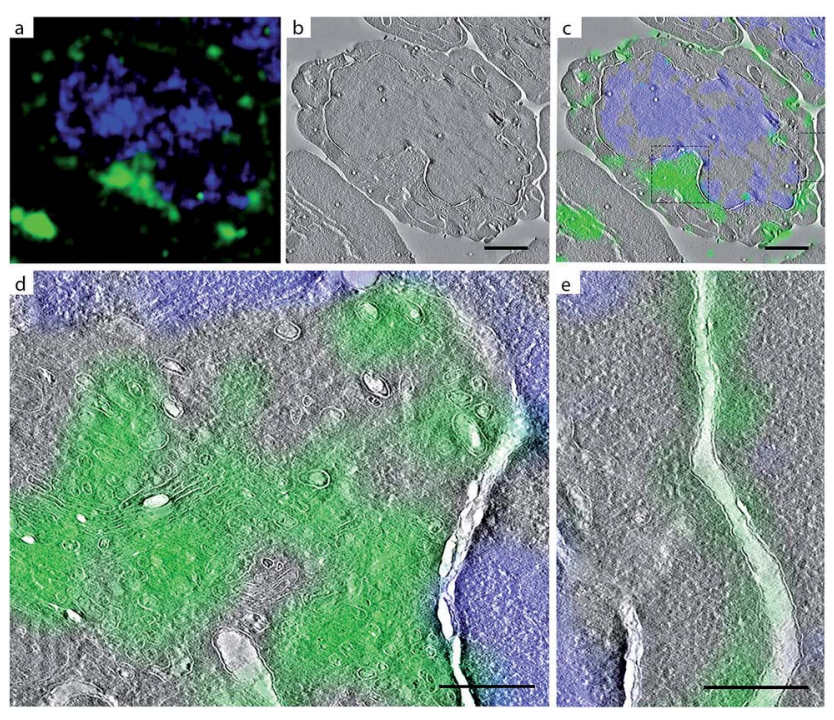

Fig. 5 CLEM imaging of bioorthogonal tagged sialylated glycans in Jurkat cells. Green: AlexaFluor-488 alkyne; blue: DAPI. DAPI staining was used for correlation purposes. (a) Confocal microscopy images; (b) EM image; (c) CLEM image obtained from overlay LM and EM pictures; (e) CLEM detail from (c) of intracellular region; CLEM detail from (c) of plasma membrane. Scale bar $2 \mu \mathrm{m}$. 

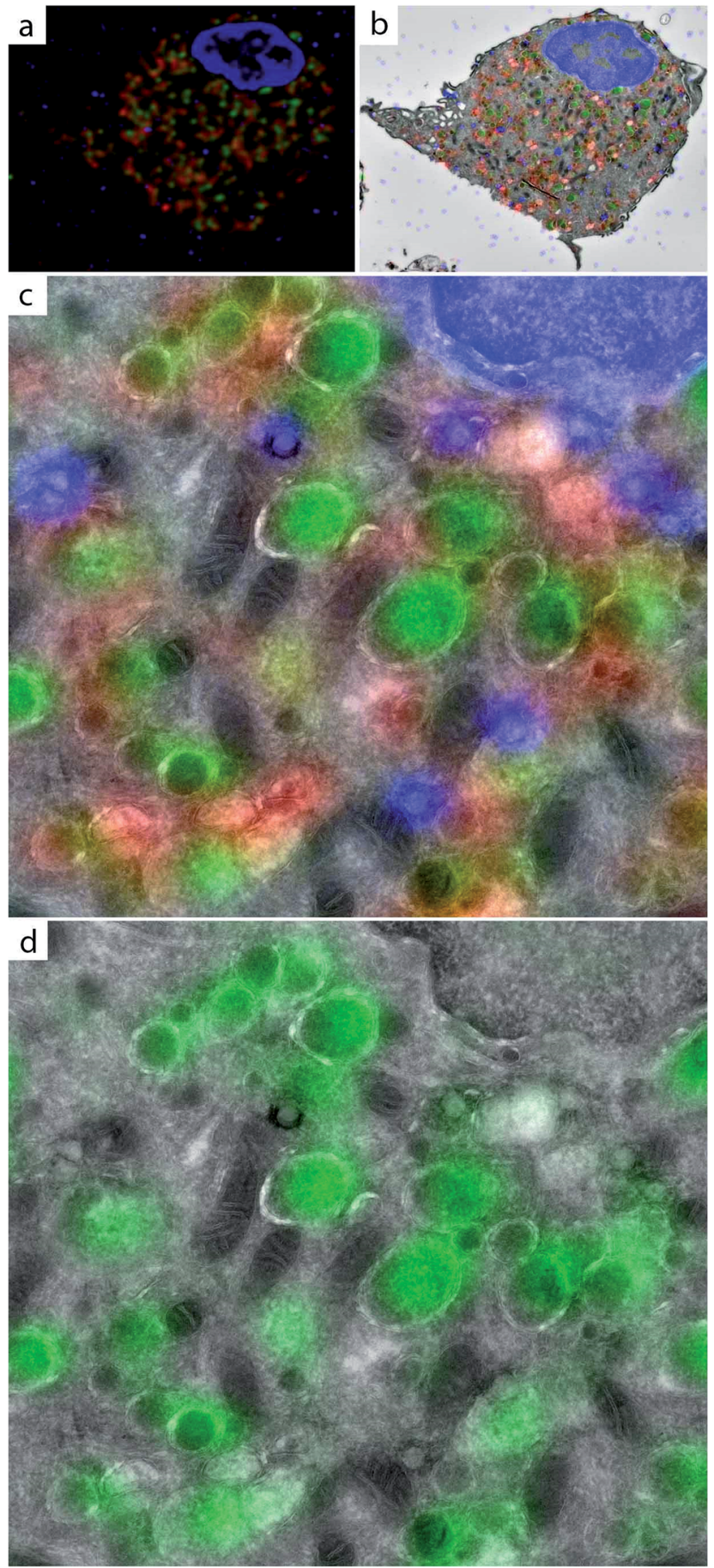

Fig. 6 CLEM imaging of active populations of cysteine proteases in BM-DCs. BM-DCs were incubated for $2 \mathrm{~h}$ with $10 \mu \mathrm{M}$ DCG-04-azide $(a-d)$. Cells were fixed in $2 \%$ PFA, subjected to Tokuyasu sample preparation and cryosectioned into $150 \mathrm{~nm}$ sections. Sections were reacted with AlexaFluor-488 alkyne using $\mathrm{ccHc}$-conditions (green), anti-LAMP-1 (red) and DAPI (blue). DAPI staining and blue fiducials were used for correlation purposes. (a) Confocal microscopy image; (b) CLEM image obtained from overlay LM and EM pictures; (c and d) CLEM details from (b), showing LAMP-1, AlexaFluor-488 and DAPI (c) or only AlexaFluor-488 (d). Scale bar $1 \mu \mathrm{m}$. in the first step enzymes are covalently and irreversibly inhibited, after which the reporter moiety is ligated in a second step through bioorthogonal chemistry. One example that was of particular interest to $\mathrm{us}^{58,59}$ were the endo-lysosomal cysteine protease family: the cathepsins ${ }^{55,60}$ as these enzymes are not only covalently labelled by a series of analogues of the natural product E64 (ref. 61) and their activity is also restricted to the endo-lysosomal pathway ${ }^{62}$ (although their extracellular activity has also been reported ${ }^{63}$ ). If their intracellular activity could be pinpointed to LAMP-1 negative vesicles in DCs, this would provide further visual evidence for the degradation of bacteria in LAMP-1-negative phagosomes observed earlier.

As such, their imaging using an azide-modified variant of E64 (DCG-04-azide) will offer an 'internal standard' of the selectivity of this approach: the signal of the enzyme is expected to be contained only within membrane-limited vesicles. Furthermore, it should in part overlap with the lysosomal marker LAMP-1.

We incubated BM-DCs with DCG-04-azide using conditions described $^{64}$ and after extensive washing (the 2-step probe is cell permeable and unbound ABP can therefore be removed prior to bioorthogonal labelling and imaging), the cells were fixed, sectioned and subjected to bioorthogonal labelling. The resulting fluorescent signal was found to be fully contained in membrane-limited structures (Fig. 6, S10 $\dagger$ ) and indeed costaining with the anti-LAMP-1 antibody revealed a population of vesicles negative for LAMP-1 but with robust cathepsin activity.

These two examples show that CLEM-imaging of bioorthogonal handles is a versatile technique that can be used over a wide range of applications. In future, it will be interesting to see how the structural information will impact the application of activity-based probes for other enzymes and enzyme families, such as the aforementioned serine hydrolases ${ }^{65}$ and the recently reported protease-specific probes. ${ }^{66}$

\section{Conclusions}

By combining BONCAT of bacteria with CLEM-imaging, we have established a new approach that allowed us to visualise bioorthogonally modified bacteria in an ultrastructural cellular context, even during late stages of bacterial degradation. This approach is of great interest for the study of obligate intracellular parasites that are very hard to study by any other means. As the application of bioorthogonal chemistry is ever expanding, the CLEM-imaging method of bioorthogonal groups described here could also be of great benefit to the study of labelled biomolecules in other fields in which bioorthogonal imaging has proven its value. ${ }^{25}$

Here we demonstrate this value by imaging glycans on the surface and in the Golgi of mammalian cells and by imaging active sub-populations of cathepsins inside the endo-lysosomal pathway. Application of this approach to other bioorthogonal assays (for instance, lipid imaging.67 and the imaging of newly synthesized proteins ${ }^{68}$ ), and perhaps in combination with some of the more recently developed bioorthogonal chemistries ${ }^{69}$ will allow the provision of additional structural information to the 
current imaging methods available for these types of biomolecules.

\section{Acknowledgements}

SvK was funded by a Starting Grant from the European Research Council (ERC-Grant number 639005). We thank Ramon A. van der Valk and Remus T. Dame for providing the GFP-plasmid.

\section{Notes and references}

1 D. M. Walker, S. Oghumu, G. Gupta, B. S. McGwire, M. E. Drew and A. R. Satoskar, Cell. Mol. Life Sci., 2014, 71, 1245-1263.

2 M. B. Johnson and A. K. Criss, J. Visualized Exp., 2013, 50729. 3 R. W. Truman and J. L. Krahenbuhl, Int. J. Lepr. Other Mycobact. Dis., 2001, 69, 1-12.

4 O. Lamrabet and M. Drancourt, Tuberculosis, 2012, 92, 365376.

5 O. V. Vieira, R. J. Botelho and S. Grinstein, Biochem. J., 2002, 366, 689-704.

6 H. Katayama, A. Yamamoto, N. Mizushima, T. Yoshimori and A. Miyawaki, Cell Struct. Funct., 2008, 33, 1-12.

7 R. K. Lim and Q. Lin, Chem. Commun., 2010, 46, 1589-1600.

8 H. C. Kolb, M. G. Finn and K. B. Sharpless, Angew. Chem., Int. Ed. Engl., 2001, 40, 2005-2021.

9 E. M. Sletten and C. R. Bertozzi, Angew. Chem., Int. Ed. Engl., 2009, 48, 6974-6998.

10 C. P. Ramil and Q. Lin, Chem. Commun., 2013, 49, 1100711022.

11 G. W. Liechti, E. Kuru, E. Hall, A. Kalinda, Y. V. Brun, M. van Nieuwenhze and A. T. Maurelli, Nature, 2014, 506, 507-510.

12 P. Shieh, M. S. Siegrist, A. J. Cullen and C. R. Bertozzi, Proc. Natl. Acad. Sci. U. S. A., 2014, 111, 5456-5461.

13 M. S. Siegrist, S. Whiteside, J. C. Jewett, A. Aditham, F. Cava and C. R. Bertozzi, ACS Chem. Biol., 2012, 8, 500-505.

14 D. C. Dieterich, A. J. Link, J. Graumann, D. A. Tirrell and E. M. Schuman, Proc. Natl. Acad. Sci. U. S. A., 2006, 103, 9482-9487.

15 P. Landgraf, E. R. Antileo, E. M. Schuman and D. C. Dieterich, Methods Mol. Biol., 2015, 1266, 199-215.

16 K. L. Kiick, E. Saxon, D. A. Tirrell and C. R. Bertozzi, Proc. Natl. Acad. Sci. U. S. A., 2002, 99, 19-24.

17 K. L. Kiick and D. A. Tirrell, Tetrahedron, 2000, 56, 94879493.

18 R. Hatzenpichler, S. Scheller, P. L. Tavormina, B. M. Babin, D. A. Tirrell and V. J. Orphan, Environ. Microbiol., 2014, 16, 2568-2590.

19 J. B. Pawlak, G. P. P. Gential, T. J. Ruckwardt, J. S. Bremmers, N. J. Meeuwenoord, F. A. Ossendorp, H. S. Overkleeft, D. V. Filippov and S. I. van Kasteren, Angew. Chem., Int. Ed. Engl., 2015, 54, 5628-5631.

20 S. P. Ouellette, F. C. Dorsey, S. Moshiach, J. L. Cleveland and R. A. Carabeo, PLoS One, 2011, 6, e16783.

21 N. van der Wel, D. Hava, D. Houben, D. Fluitsma, M. van Zon, J. Pierson, M. Brenner and P. J. Peters, Cell, 2007, 129, 1287-1298.
22 P. de Boer, J. P. Hoogenboom and B. N. G. Giepmans, Nat. Methods, 2015, 12, 503-513.

23 I. Nikić, T. Plass, O. Schraidt, J. Szymański, J. A. G. Briggs, C. Schultz and E. A. Lemke, Angew. Chem., Int. Ed. Engl., 2014, 53, 2245-2249.

24 R. S. Erdmann, H. Takakura, A. D. Thompson, F. RiveraMolina, E. S. Allgeyer, J. Bewersdorf, D. Toomre and A. Schepartz, Angew. Chem., Int. Ed. Engl., 2014, 53, 1024210246.

25 D. M. van Elsland, E. Bos, H. S. Overkleeft, A. J. Koster and S. I. van Kasteren, J. Chem. Biol., 2015, 1-5.

26 B. N. G. Giepmans, Histochem. Cell Biol., 2008, 130, 211-217.

27 E. M. Sletten and C. R. Bertozzi, Acc. Chem. Res., 2011, 44, 666-676.

28 C. P. Ramil and Q. Lin, Chem. Commun., 2013, 49, 1100711022.

29 D. J. Leahy, W. A. Hendrickson, I. Aukhil and H. P. Erickson, Science, 1992, 258, 987-991.

30 W. B. Wood, J. Mol. Biol., 1966, 16, 118-133.

31 A. K. Upadhyay, A. Murmu, A. Singh and A. K. Panda, PLoS One, 2012, 7, e33951.

32 K. T. Tokuyasu, J. Cell Biol., 1973, 57, 551-565.

33 C. W. Tornøe, C. Christensen and M. Meldal, J. Org. Chem., 2002, 67, 3057-3064.

34 V. V. Rostovtsev, L. G. Green, V. V. Fokin and K. B. Sharpless, Angew. Chem., Int. Ed. Engl., 2002, 41, 2596-2599.

35 M. Lo Conte, S. Staderini, A. Marra, M. Sanchez-Navarro, B. G. Davis and A. Dondoni, Chem. Commun., 2011, 47, 11086-11088.

36 L. I. Willems, N. Li, B. I. Florea, M. Ruben, G. A. van der Marel and H. S. Overkleeft, Angew. Chem., Int. Ed., 2012, 51, 4431-4434.

37 V. Hong, N. F. Steinmetz, M. Manchester and M. G. Finn, Bioconjugate Chem., 2010, 21, 1912-1916.

38 W. Kukulski, M. Schorb, S. Welsch, A. Picco, M. Kaksonen and J. A. Briggs, J. Cell Biol., 2011, 192, 111-119.

39 C. L. Thomas and A. J. Maule, J. Gen. Virol., 2000, 81, 18511855.

40 U. Schnell, F. Dijk, K. A. Sjollema and B. N. G. Giepmans, Nat. Methods, 2012, 9, 152-158.

41 G. Griffiths and J. M. Lucocq, Histochem. Cell Biol., 2014, 142, 347-360.

42 M. B. Lutz, N. Kukutsch, A. L. J. Ogilvie, S. Rößner, F. Koch, N. Romani and G. Schuler, J. Immunol. Methods, 1999, 223, 77-92.

43 M. A. West, R. P. A. Wallin, S. P. Matthews, H. G. Svensson, R. Zaru, H. G. Ljunggren, A. R. Prescott and C. Watts, Science, 2004, 305, 1153-1157.

44 L.-P. Erwig, K. A. McPhilips, M. W. Wynes, A. Ivetic, A. J. Ridley and P. M. Henson, Proc. Natl. Acad. Sci. U. S. A., 2006, 103, 12825-12830.

45 E.-Y. Seo, T.-S. Ahn and Y.-G. Zo, Appl. Environ. Microbiol., 2010, 76, 1981-1991.

46 A. Savina, C. Jancic, S. Hugues, P. Guermonprez, P. Vargas, I. C. Moura, A.-M. Lennon-Duménil, M. C. Seabra, G. Raposo and S. Amigorena, Cell, 2006, 126, 205-218. 
47 I. Cebrian, G. Visentin, N. Blanchard, M. Jouve, A. Bobard, C. Moita, J. Enninga, L. F. Moita, S. Amigorena and A. Savina, Cell, 2011, 147, 1355-1368.

48 O. P. Joffre, E. Segura, A. Savina and S. Amigorena, Nat. Rev. Immunol., 2012, 12, 557-569.

49 E. Saxon and C. R. Bertozzi, Science, 2000, 287, 2007-2010.

50 A. E. Speers, G. C. Adam and B. F. Cravatt, J. Am. Chem. Soc., 2003, 125, 4686-4687.

51 R. M. Werner, L. M. Williams and J. T. Davis, Tetrahedron Lett., 1998, 39, 9135-9138.

52 J. M. Baskin, J. A. Prescher, S. T. Laughlin, N. J. Agard, P. V. Chang, I. A. Miller, A. Lo, J. A. Codelli and C. R. Bertozzi, Proc. Natl. Acad. Sci. U. S. A., 2007, 104, 16793-16797.

53 B. F. Cravatt, A. T. Wright and J. W. Kozarich, Annu. Rev. Biochem., 2008, 77, 383-414.

54 L. I. Willems, H. S. Overkleeft and S. I. van Kasteren, Bioconjugate Chem., 2014, 25, 1181-1191.

55 D. Greenbaum, K. F. Medzihradszky, A. Burlingame and M. Bogyo, Chem. Biol., 2000, 7, 569-581.

56 L. E. Sanman and M. Bogyo, Annu. Rev. Biochem., 2014, 83, 249-273.

57 H. Ovaa, P. F. van Swieten, B. M. Kessler, M. A. Leeuwenburgh, E. Fiebiger, A. M. van den Nieuwendijk, P. J. Galardy, G. A. van der Marel, H. L. Ploegh and H. S. Overkleeft, Angew. Chem., Int. Ed. Engl., 2003, 42, 3626-3629.
58 S. I. van Kasteren, I. Berlin, J. D. Colbert, D. Keane, H. Ovaa and C. Watts, ACS Chem. Biol., 2011, 6, 1198-1204.

59 S. I. van Kasteren and H. S. Overkleeft, Curr. Opin. Chem. Biol., 2014, 23, 8-15.

60 V. Turk, V. Stoka, O. Vasiljeva, M. Renko, T. Sun, B. Turk and D. Turk, Biochim. Biophys. Acta, Proteins Proteomics, 2012, 1824, 68-88.

61 A. J. Barrett, A. A. Kembhavi, M. A. Brown, H. Kirschke, C. G. Knight, M. Tamai and K. Hanada, Biochem. J., 1982, 201, 189-198.

62 C. Watts, Biochim. Biophys. Acta, Proteins Proteomics, 2012, 1824, 14-21.

63 J. S. Mort, A. D. Recklies and A. R. Poole, Arthritis Rheum., 1984, 27, 509-515.

64 S. Hoogendoorn, G. H. van Puijvelde, J. Kuiper, G. A. van der Marel and H. S. Overkleeft, Angew. Chem., Int. Ed. Engl., 2014, 53, 10975-10978.

65 Y. Liu, M. P. Patricelli and B. F. Cravatt, Proc. Natl. Acad. Sci. U. S. A., 1999, 96, 14694-14699.

66 L. E. Edgington, M. Verdoes and M. Bogyo, Curr. Opin. Chem. Biol., 2011, 15, 798-805.

67 H. C. Hang, J. P. Wilson and G. Charron, Acc. Chem. Res., 2011, 44, 699-708.

68 K. E. Beatty, J. C. Liu, F. Xie, D. C. Dieterich, E. M. Schuman, Q. Wang and D. A. Tirrell, Angew. Chem., Int. Ed. Engl., 2006, 45, 7364-7367.

69 K. Lang and J. W. Chin, ACS Chem. Biol., 2014, 9, 16-20. 